Absence makes the mind grow sharper: Effects of element omission on subseque...

The authors investigate the memorial consequences of deliberately omitting crucial elements from an advertisement. Research on the selfgeneration effect in cognitive psychology indicates that such element omission may actually lead to an improvement in recall. Support for this perspective is obtained in a series of experiments that explores the effects of feature omission in the context of both overt omission (in which the omission is highlighted by the advertiser) and implicit omission (in which the omission, though not highlighted in the advertisement, is noticed because of prior expectations for that ad type). Even under highly constrained processing conditions (e.g., exposure times as short as four seconds), an advertisement that omits a key element is shown to produce better recall than an equivalent advertisement that contains the element. The authors find that this recall improvement occurs along dimensions that are specifically related to the omitted element; therefore, leaving out an element related only to the product category (but not the brand) produces an improvement only in category recall, whereas brand recall is improved by the omission of an element related to the brand name. The authors discuss theoretical and managerial implications of these findings.

\title{
Absence Makes the Mind Grow Sharper: Effects of Element Omission on Subsequent Recall
}

Although it seems obvious that advertisements should present complete information, advertisers often omit important elements from their advertisements. Many cases of such element omission can be found: A survey has shown that $44 \%$ of advertisements contain an omission of one type or another (Faier and Unger 1987; see also Aaker, Batra, and Myers 1992; Peracchio and Meyers-Levy 1994). Advertisers may choose to omit certain elements for strategic reasons. For example, some advertisements compare two brands but omit an explicit conclusion regarding the superiority of the advertised brand, leaving the viewers to generate the conclusion themselves (Johar 1995; Kardes 1988; Sawyer and Howard 1991; Stayman and Kardes 1992). Other advertisements highlight omissions even more overtly by using incomplete words or pictures. For example, an advertise-

*Jaideep Sengupta is Assistant Professor of Marketing and Wei Lun Senior Fellow (e-mail: mkjaisen@ust.hk), and Gerald J. Gom is Professor of Marketing (e-mail: mkgorn @ust.hk), School of Business and Management, Hong Kong University of Science and Technology. The authors thank the three anonymous $J M R$ reviewers for their valuable input. Frank Kardes and Akshay Rao provided insightful comments on previous versions of this article. The financial assistance offered by the Hong Kong Research Grants Council is gratefully acknowledged. ment for J\&B scotch whisky features the tag line "...ingle ells, ... ingle ells.... The holidays aren't the same without J\&B." Similarly, a significant portion of a visual outline may sometimes be cropped, as in an advertisement for Bugle Boy jeans that shows only the lower portion of a man's jean-clad legs entwined with the back of a woman's legs (Peracchio and Meyers-Levy 1994). Omissions may also be driven by policy concerns - for example, advertisements for alcohol products often portray people either just before or just after alcohol consumption. Although advertisers want viewers to generate consumption-related inferences, regulations prohibit showing actual consumption. On a related front, legislation in the United States and European Economic Community (EEC) now bans the use of animated characters (e.g., Joe Camel) in cigarette commercials. In the EEC, human figures (e.g., the Marlboro man) are banned as well, and anti-tobacco-advertising advocates in the United States have argued for a similar policy.

These examples illustrate that the omission of crucial ad elements is a fairly common phenomenon in advertising. The current research explores the memory-related consequences (e.g., product category recall, brand recall) of this strategy. Such an investigation is important for practical as well as theoretical reasons. From a theoretical perspective, 
an examination of the recall effects of element omission in advertising provides a deeper understanding of the cognitive processes related to and arising from the "completion" of incomplete/missing items (see Slamecka and Graf 1978). From a manager's viewpoint, the study of recall effects is important because the high level of clutter that exists in today's marketplace makes advertising recall a goal that is both sought after and difficult to achieve (Aaker, Batra, and Myers 1992). In many situations, particularly for lowinvolvement packaged goods, it can be argued that the recall achieved by an advertisement is at least as important as the degree of brand liking induced, because recall is a critical predictor of purchase in the low-involvement hierarchy of communication effects (Krugman 1965; Ray 1982). Accordingly, by focusing on recall-related outcomes of element omission in advertising, we address an issue that is important not only to researchers but to practitioners as well.

In light of the tenet that viewers typically process the visual elements of advertising first (Houston, Childers, and Heckler 1987; Mitchell 1983), we focus on the recall effects of leaving out a key visual element from an advertisement. Visual element omission may be created in two broad ways. In the first case, the advertiser highlights the omissionsuch as when the missing element is explicitly outlined in the advertisement. For example, the central feature of a recent advertisement for Olympus cameras depicts the outline of a strap hanging around a man's neck, at the end of which is the outline of a camera on his chest (see Appendix A, Part I). Although most viewers would be likely to realize that the outline is supposed to represent a camera, this crucial product-relevant element has been deliberately and overtly omitted by the advertiser. The label we use for cases such as these, in which the omission/incompleteness is clearly highlighted by the advertiser, is "overt omission."

In another type of visual element omission, the advertiser may not overtly highlight the absence of the element; indeed, there may be no actual "incompleteness" in the advertisement. Even so, prior expectations may lead viewers to perceive an omission. In particular, consumers often have well-formed ad schemas for highly advertised products (Goodstein 1993; Goodstein, Moore, and Cours 1992), including a knowledge of the important elements they typically expect to see in an ad campaign for a particular brand (e.g., consumers would usually expect to see a cowboy-the Marlboro Man-in an advertisement for Marlboro cigarettes). If one of these elements is left out, consumers are likely to notice the omission, even when it is not overtly brought to their attention. We label cases such as these, in which the omission is likely to be implicitly inferred by consumers even when it is not highlighted by the advertiser, "implicit omission."1

Although the previous two cases differ in several respects, they share an important similarity -in both cases, an important element is omitted from the advertisement. The major goal of this article is to examine whether these different forms of element omission can improve later recall. We also

\footnotetext{
IWe note that the distinction between the two types of omissions may also be captured by other terms; the terminology used here is simply intended for expository purposes rather than for the presentation of a comprehensive typology of omission types. Note also that the term "implicit omission" in this context bears no relation to implicit memory effects (e.g., Schacter 1987)
}

examine whether such an effect may be obtained even when the advertisement is processed in a cursory manner. Prior research has shown that recall can be improved when consumers engage in a significant amount of ad processing (Goodstein 1993; Petty, Cacioppo, and Schumann 1983). These findings, though of undoubted importance, may be less applicable in situations in which consumers do not spend much time or effort in viewing an advertisement, as is typically the case in nonlaboratory settings. Indeed, it is estimated that only $20 \%$ of print advertisements are read even a little, and very few are read comprehensively (Burnett and Moriarty 1998). Therefore, it is important to examine whether recall can be increased even with "thin slice" processing (Ambady, Bernieri, and Richeson 2000), such as when consumers view the advertisement for a very short time.

Drawing on research in the area of cognitive psychology, we suggest that the absence of key ad elements might increase recall along specific dimensions, even under relatively constrained conditions. We also seek to provide insights into the mechanisms underlying our results, thus adding to the extant theoretical knowledge on the wellknown generation effect in cognitive psychology, which posits that items that are generated by participants themselves are remembered better than items provided in a complete form (Slamecka and Graf 1978). In particular, our findings address the debate on whether the generation effect is simply an artifact of the greater distinctiveness of incomplete items (Slamecka and Katsiati 1987) or whether the effect occurs because of substantive psychological processes associated with self-generation (Gardiner 1988; Nairne and Widner 1988).

The discussion that follows primarily pertains to the case of overt omission, as exemplified by the Olympus advertisement described previously (and presented in Appendix A, Part I). Subsequently, we extend our arguments to implicit omission as well.

\section{THEORETICAL BACKGROUND}

Researchers in cognitive psychology have found abundant evidence for the self-generation effect on several different memory tasks, including cued recall, free recall, and recognition (Jacoby 1983; McElroy and Slamecka 1982; Payne, Neely, and Burns 1986; Slamecka and Graf 1978). For example, Gardiner (1988) asked participants to generate a target word on the basis of the word fragment and a semantic clue to the meaning of the word (e.g., "assa---n," paired with "political killer"). Participants in this "generate" condition performed better on a subsequent memory test of target words than did participants who were asked to read the complete version of the target word (along with the cue) at exposure.

The self-generation effect has direct implications for the memory consequences of element omission, particularly when considered in light of prior advertising research on the effects of incomplete stimuli (Peracchio and Meyers-Levy 1994; Sawyer and Howard 1991; Stayman and Kardes 1992). Although this research has examined attitude effects rather than recall, its relevance to the current focus lies in its underlying premise regarding the spontaneous generation of missing ad elements. Specifically, research streams on openended advertising (in which an explicit conclusion is delib- 
erately omitted from the advertisement; Sawyer and Howard 1991) and object-cropping (i.e., cutting off a significant portion of the outline of a visual element; Peracchio and Meyers-Levy 1994) both base their persuasion findings on the implicit assumption that consumers can themselves generate the missing element in an advertisement, without external prompting. ${ }^{2}$ Thus, the attitude improvement induced by open-ended advertisements is assumed to be a function of the greater credibility of self-generated arguments (versus externally provided ones), and one reason for the improved attitudes produced by object cropping is held to be the positive affect produced by "completing" the cropped object (Kreitler and Kreitler 1972).

In the current context, the notion that viewers engage in such spontaneous closure suggests that highlighting the absence of an ad element (e.g., by outlining a camera on the man's chest instead of showing the camera itself) will lead viewers to think of the missing element themselves. According to the self-generation literature, voluntary retrieval of the element (compared with being presented with the complete element) should result in improved recall. In other words, better recall should be obtained when a key visual element is absent from the advertisement than would be obtained if the visual element were presented in toto.

Exactly how does this recall improvement take place? This is an important question for theoretical reasons and because a knowledge of the underlying process enables us to predict the precise nature of the recall improvement that might be obtained by element omission. Explanations offered for the generation effect can be divided into two broad categories. One class of explanations asserts that the effect arises because of differences in the psychological mechanisms associated with generating an element versus processing the same element when it is provided externally. Although there are some subtle differences in the various explanations within this category (e.g., Gardiner 1988; McDaniel, Waddill, and Einstein 1988; McFarland, Frey, and Rhodes 1980), a common view is that generating an item results in greater activation of that item's conceptual meaning, which produces better retention (McElroy and Slamecka 1982; Nairne, Pusen, and Widner 1985; Nairne and Widner 1988). Note that the increased semantic activation produced by item generation is held to be narrowly restricted to the generated item itself and does not include the surrounding context (Schmidt and Cherry 1989; Slamecka and Graf 1978). For example, Slamecka and Graf (1978) found significantly improved memory performance for target words that were self-generated in response to cue words (rather than provided explicitly by the experimenter); however, this generation effect did not result in improved memory for the cue words themselves.

This category of explanations can therefore be classed under the general label of "specific activation" (in reference to the greater activation of the generated element). Applied to the advertising context, the specific activation mechanism

2For both open-ended advertising and object cropping, such self-generation (and thus, the improvement in attitudes) is held to be more likely under conditions of high motivation. However, clear, unambiguous cases of incompleteness (such as those studied in this research) create an intrinsic pressure toward completion that is difficult to resist (Kreitler and Kreitler 1972); accordingly, an external motivational inducement should not always be necessary for viewers to engage in generation. suggests that omitting an ad element should improve later recall specifically along dimensions related to that element. For example, in the case of the Olympus advertisement that exemplifies overt omission, the omitted element (i.e., a missing camera on the man's chest) is related only to the product category (camera). Therefore, according to the specific activation mechanism, generation of this element should yield an improvement only in product category recall (without influencing recall for other features such as brand name and ad content).

Our research favors the specific activation mechanism as an explanation for the generation effect-in other words, we lean toward the view that there is indeed a substantive difference between generating an item and processing it in a complete format. However, an alternative viewpoint must also be considered. In contrast to the activation hypothesis, this class of explanations suggests that there is nothing special about the generation process per se that leads to improved recall for items that are presented in some sort of incomplete format, such as a word fragment (Begg and Snider 1987; Slamecka and Katsiati 1987). On the basis of the observation that generation effects have most often been reported in within-subjects experimental designs in which participants are exposed to both incomplete and complete items, Slamecka and Katsiati (1987) have suggested that incomplete items, because of their cryptic and fragmentary nature, are merely more distinctive. That is, incomplete items in a list simply stand out more than complete items and are therefore recalled better. In support of this conclusion, these authors found no evidence of a generation effect in a between-subjects design in which participants were exposed to only incomplete words or only complete words, though the generation effect was observed when the researchers used the same stimuli in a within-subjects design (for similar results, see Begg and Snider 1987).

An application of this logic in the current context suggests that improved recall may be obtained for an advertisement in which a crucial element is outlined rather than provided in toto, simply because such a strategy makes the advertisement distinctive and unusual (especially when viewed in the context of other advertisements that do not contain such incompleteness). In other words, the predicted improvement in recall arising from element omission may have nothing to do with the specific activation/generation of the missing element. It is not even necessary that the missing element be generated for recall improvement to occur; rather, recall may simply improve because of the distinctiveness of the advertisement itself. This distinctivenessbased mechanism thus argues that a strategy of omission (e.g., presenting only the outline of an element), because it makes the advertisement stand out more, can lead to improved recall irrespective of whether the missing element has any relevance to the advertised product. Note that this view contrasts with the specific activation mechanism, according to which a product-irrelevant omission should not produce any improvement in recall, because such an omission will not lead to the generation (and consequent activation) of a product-relevant concept.

In further contrast to the specific activation mechanism, which argues that recall improvement is restricted to the missing element (e.g., only improved product category recall for the Olympus advertisement under discussion), the distinctiveness explanation suggests that because element 
omission makes the advertisement itself distinctive, recall improvement should be obtained for the advertisement as a whole (including the brand name and other ad content). Indeed, such a result would be consistent with previous advertising research, which has found that novel/atypical advertisements produce better overall recall (Goodstein 1993; Houston, Childers, and Heckler 1987; Meyers-Levy and Tybout 1989). For example, Goodstein (1993) found that an atypical advertisement for a shampoo (featuring an interview with a foreign exchange student) led to better recall than a typical version of the advertisement (featuring a woman washing her hair). More important, the recall improvement thus obtained extended to other elements in the advertisement (e.g., attribute claims) apart from the distinctive item itself. The rationale for this and similar findings (e.g., Houston, Childers, and Heckler 1987) is that an unexpected/distinctive element in an advertisement leads to greater overall processing of the ad content, as the viewer attempts to make sense of the entire set of information in the advertisement and resolve any incongruity it may contain. In turn, such overall elaboration (even if it does not always result in successful incongruity resolution, especially for extreme forms of atypicality) results in a widespread improvement in ad recall (Houston, Childers, and Heckler 1987; Meyers-Levy and Tybout 1989).

Relatedly, the two mechanisms (specific activation and distinctiveness) can be further differentiated on the basis of the moderating impact of processing opportunity. Specifically, the elaboration of an entire set of stimulus features (as dictated by the distinctiveness hypothesis) should require greater cognitive resources than the narrower focus on a single element that is required by specific activation. Accordingly, the former process is more likely to be constrained by the opportunity to process, as operationalized by factors such as the amount of ad viewing time (Houston, Childers, and Heckler 1987; Srull 1981). For example, Houston, Childers, and Heckler (1987) found that the improvement in ad recall obtained as a result of increased ad elaboration (produced by the deviation of advertising copy from pictureinduced expectations) disappeared when ad exposure time was reduced from 15 seconds to 10 seconds. In contrast, lowering the opportunity to process should be less likely to affect the recall improvement obtained by the less resourceintensive process of specific activation.

Our research not only seeks to study whether element omission in advertising leads to any improvement in recall but also investigates the mechanism underlying the obtained recall findings and provides further insights into the oftendocumented generation effect. Specifically, we aim to show that the effect arises because of substantive differences between the psychological mechanisms associated with generating an item and those associated with processing that item in a complete form and that the effect is not simply a distinctiveness-induced artifact. Thus, we suggest that element omission can lead to recall improvement even when the role of distinctiveness-induced elaboration is minimized. Not only is this an important point for theoretical reasons, but it also has practical consequences in the advertising context in terms of whether recall improvement is specific or general and whether the recall effect can be obtained under constrained processing conditions.

Experiment $I$ investigates these issues in the context of overt omission, and Experiment 2 examines a case of implicit omission. The overall pattern of results in each of these experiments was supportive of the specific activation process, though participants in these experiments were not explicitly asked to generate the missing element because of the relatively intrusive nature of such a procedure (see Kardes 1988). More direct support for the specific activation mechanism was provided by follow-up experiments (Experiments $1 \mathrm{~A}$ and $2 \mathrm{~A}$ ) that explicitly instructed participants to generate the missing element during exposure to the ad. Furthermore, although the major focus of this research is recall effects, these follow-up experiments also included attitude measures to broaden the scope of our investigation into the effects of element omission. ${ }^{3}$

\section{EXPERIMENT I}

A total of 118 business undergraduate students participated in a 2 (ad type: element-absent/element-present) $\times 2$ (exposure time: 4 seconds $/ 8$ seconds) +1 (control advertisement: product-unrelated omission/8-second exposure) between-subjects experiment, which examined whether the overt omission of a key ad element would lead to any improvement in recall compared with an advertisement that contained the element.

\section{Stimuli and Manipulations}

Test advertisement. A real print advertisement for Olympus cameras was used as the test advertisement. The advertisement featured a man's bare-chested upper torso. In the element-absent (incomplete) version of the advertisement, the outline of a camera strap and camera was displayed on the man's chest (see Appendix A, Part I). The bottom portion of the advertisement contained the following items: (1) a slogan, "The official cameras of summer"; (2) pictures of four small cameras; (3) general text related to product attributes (e.g., "extremely easy to use"); and (4) the brand name in bold white lettering.

To create the element-present (complete) version of the advertisement, the outline of the strap and the camera on the man's chest were professionally filled in using Photoshop software (Appendix A, Part II). Thus, the element-absent version of the advertisement featured an overt omission of a product-relevant element (i.e., the camera on the man's chest), whereas the element-present version of the advertisement contained the camera.

In addition to these two versions of the test advertisement, we also created a control advertisement in which, as in the element-absent condition, we again sought to create a prominent and noticeable omission by presenting an outline on the man's chest. However, instead of an outline in a rectangular camera shape (product-related), the outline in the control condition was of a product-unrelated circular form, of about the same area as the rectangular outline (Appendix A, Part III). This control advertisement helped provide insights into the mechanism underlying the predicted recall effects of element omission. According to the distinctiveness mechanism, the outlining technique, by virtue of its atypicality, simply makes the test advertisement distinctive (and therefore more memorable) compared with the filler adver-

3The follow-up experiments were conducted together, after the two main studies had been run. However, as suggested by a reviewer, we discuss each of the follow-ups along with the respective main experiment for expository purposes. 
tisements. Such a rationale argues that any advertisement that features a highlighted outline (rather than a complete, filled-in visual) should lead to improved recall, even if the element outlined is not product related. Conversely, if the predicted improvement in recall for the element-absent test advertisement is obtained because of greater activation of a product-related node, we would not expect a similar recall improvement for the control advertisement, which features a product-unrelated outline.

Pretesting was carried out to check that the rectangular outline in the element-absent test advertisement, but not the circular outline for the control advertisement, would lead participants to think of a camera. Twenty-eight participants were shown the incomplete advertisement (depicting the camera outline) on an overhead projector (OHP). The experimenter (an assistant unconnected with the research) then pointed to the outline and asked, "What is the first thing that comes to mind when you see this? Please write down your answer in the space provided." Twenty-five participants $(89.28 \%)$ wrote down "camera" as their first reaction, lending support to the idea that viewing the outline would indeed lead participants to think of the product category. In contrast, not one of another group of participants $(n=39)$ who was asked the same question for the control advertisement responded with "camera." The modal answer for this group was "medal"; this response was listed by only 8 participants. Thus, as intended, element omission in the control advertisement did not appear to induce inferences related to the product category.

Exposure times. Depending on the condition, subjects were exposed to all advertisements in the main study for a period of 8 seconds or 4 seconds each. If, as is suggested by the distinctiveness hypothesis, element omission improves recall by increasing overall elaboration of the entire advertisement, we would expect these recall differences to be eliminated (or at least substantially diminished) when exposure time is significantly lowered (Houston, Childers, and Heckler 1987). However, if the relatively effortless specific activation process (which only requires increased activation of a single element) is sufficient for improved recall, lowering the opportunity to process is less likely to affect recall results.

We therefore needed two levels of exposure to check whether the predicted influence of element omission on recall would be moderated by exposure time. We chose the specific exposure times (8-second and 4-second) on the basis of pretesting and the goals of the current research. In particular, we deliberately chose lower times than have been used in prior research that also manipulates exposure time to examine the mechanism underlying improved ad recall ( 15 and 10 seconds; Houston, Childers, and Heckler 1987), for applied as well as experimental reasons. From an applied viewpoint, the use of low exposure times fits with our goal of documenting recall improvement under conditions that mirror real-life situations, in which consumers typically study advertisements for very short durations (Burnett and Moriarty 1998). From a theory-testing perspective, the test advertisement we used was relatively sparse compared with the test advertisement used in Houston, Childers, and Heckler's (1987) research. Thus, a ceiling effect on recall might have been obtained had we used relatively high times- that is, equivalently high recall could have resulted for both the element-absent and the element-present advertisement.
Therefore, even the higher time used in the current research ( 8 seconds) was chosen to be less than the lower time ( 10 seconds) used in Houston, Childers, and Heckler's research. A $100 \%$ reduction in time was then used to fix the lower time of 4 seconds to ensure a substantial lowering of processing opportunity. The choice of 4 seconds as the lower exposure time also has a precedent in prior research that has studied limited, nonelaborative processing of relatively sparse print advertisements containing a pictorial component (Childers and Houston 1984).

We note that our choice of two low exposure times introduced the possibility that participants might not be able to engage in much ad elaboration in either exposure condition. However, we judged this to be an acceptable contingency in light of the goals of the current research, which differ from those in prior research (Houston, Childers, and Heckler 1987). Specifically, Houston, Childers, and Heckler theorized that careful ad elaboration was required for recall improvement to be obtained by the particular strategy they studied (a mismatch between picture and ad copy); accordingly, enough processing opportunity had to be allowed in at least one condition ( 15 seconds) for the key recall result to be obtained. In contrast, the current research examines whether element omission might lead to recall improvement even when careful ad elaboration is not possible. Low exposure times seemed suitable from this perspective.

A pretest revealed that the two exposure times chosen, though both low in an absolute sense, were rated differently by participants in terms of processing opportunity. From our pool of undergraduate students, 48 participants were exposed to the element-absent version of the test advertisement on an OHP for either 8 or 4 seconds. They were then asked to rate whether the advertisement had been put up for enough time for them to view its details on a nine-point scale $(-4=$ "not enough time," $0=$ "just enough time," $4=$ "more than enough time"). They were also asked to indicate their agreement or disagreement with the statement, "I got enough time to view the ad details," on another nine-point scale ( 1 = "disagree," $9=$ "agree"). On both scales, the 8 second exposure (Scale 1 mean $=1.25$, Scale 2 mean $=6.58$ ) was rated higher than the 4-second exposure (Scale 1 mean $=-1.54$, Scale 2 mean $=3.46 ; p<.05$ for both scales)

\section{Procedure}

Participants were signed up through an undergraduate subject pool and were given research credit for their participation. All experimental sessions were run in the same room by an experimenter who was blind to the hypotheses. To start the experiment, participants were first asked to complete a short filler questionnaire about the prices of various brands (no camera brands were included). This was used simply as a decoy to lessen the likelihood of ceiling effects on our key recall measure. Such effects might have resulted if participants had focused all their mental resources on the advertisements that followed. After this initial questionnaire was completed, each of seven print advertisements was shown on an OHP for 8 or 4 seconds, depending on the condition (the control advertisement condition used an 8-second exposure). In each condition, the test advertisement for Olympus cameras was the second one shown. The other six advertisements, which acted as fillers and remained the same across conditions, were real print advertisements for brands such as Clorox detergent, Toyota cars, and so forth 
(none of the filler advertisements featured cameras or cigarettes, the product category studied in Experiment 2). Again, to avoid ceiling effects on recall and to simulate real-world ad viewing conditions, an incidental learning procedure was used at the time of ad exposure-that is, participants were not told that their memory for the advertisements would be tested (see Heckler and Childers 1992). Note, however, that to ensure at least a minimal level of attention, our procedure involved forced exposure to the advertisements. Although this approach follows the generation effect research in cognitive psychology (Nairne, Pusen, and Widner 1985; Slamecka and Graf 1978) and has often been used in laboratory research on advertising effects (Childers and Houston 1984; Heckler and Childers 1992; Peracchio and MeyersLevy 1994), it represents a departure from real-world conditions and to that extent limits the external validity of our findings.

After exposure to the advertisements, participants were asked to complete another decoy task, which provided a reason they had been shown the advertisements and led them to believe that the experiment had concluded. They were told that the researchers were interested in the effects of exposure to different types of stimuli on subsequent tasks and that, instead of being shown advertisements, other participants had been given some mathematical puzzles to work on (this was untrue). The researchers now wanted to see how exposure to these different stimuli would affect participants' performance on a map task. Participants were then given five minutes to draw a map of the world on a sheet of paper and label as many countries as possible.

On completion of this task, participants were asked to respond to a three-page questionnaire booklet that contained only questions about product category and brand name recall. After a cover sheet, we measured product category recall by asking participants to list all the product categories for which they had been shown advertisements a few minutes earlier. On the next page of the questionnaire, participants listed all the brands they remembered from the advertisements shown earlier. On completion of this questionnaire, a second booklet of follow-up questions was administered. Participants were first asked to write down everything they could remember from the advertisement that had been shown for Olympus cameras. Next, they were asked to write down all the thoughts they had while viewing this advertisement. The recall protocols were coded by two independent coders for the total number of distinct items recalled from the advertisement (including specific pictorial elements as well as text items); similarly, the thought protocols were coded for the total number of distinct thoughts listed. Coder agreement in each case exceeded $79 \%$, and discrepancies were resolved by an author. After completing the protocols, participants rated their familiarity with the Olympus brand and provided age and sex information; none of these items affected any of the results, and therefore the items are not discussed further.

\section{Results}

The 2 (element-present/element-absent $) \times 2(8$ seconds $/ 4$ seconds) design was analyzed with the SAS (1999-2000) procedure CATMOD for categorical variables (such as product category recall and brand recall) and analysis of variance for continuous variables such as total ad recall, number of thoughts, and so forth. Comparisons with the control condi- tion (product-unrelated element-absent advertisement) are reported subsequently.

Our chief goal was to examine whether recall would be significantly better in the element-absent condition than in the element-present condition. We tested this hypothesis by analyzing recall on three separate indicators: (1) product category recall, (2) brand name recall, and (3) total number of distinct ad elements listed on the open-ended recall measure. According to the distinctiveness perspective, element omission is likely to produce an improvement along all these dimensions. In contrast, according to the specific activation conceptualization, an improvement in recall should be obtained only along dimensions directly related to the omitted element. In this case, because the camera outline is related only to the product category, better recall should be obtained only for the product category (not for the brand name or for ad content). Furthermore, if the distinctiveness process is at work, recall improvement due to element omission is likely to be moderated by the exposure time factor; such a moderating effect is less likely to be obtained for the specific activation mechanism.

The analysis for product category recall (coded as a 1/0 variable) yielded results that supported the advantages of element omission. Ad type exerted a significant effect (element-absent recall proportion $=86.2 \%$, element-present recall proportion $=61.9 \% ; \chi^{2}=6.74, p<.01$ ). Furthermore, the interaction of ad type with exposure time was not significant $\left(\chi^{2}<1\right.$, not significant [n.s.]). The pattern of recall proportions (see Table 1) confirmed that product category recall was higher for the element-absent advertisement in the 4second exposure condition as well as the 8-second exposure condition. Thus, exposure time did not moderate the effects of element omission on category recall-a pattern of results more consistent with the specific activation mechanism than the distinctiveness mechanism.

In further support of specific activation, the recall advantage of element omission was restricted to the product category. For brand recall, neither the main effect of ad type (element-absent advertisement $=20.0 \%$, element-present advertisement $=28.21 \% ; \chi^{2}=1.05, p>.30$ ) nor the interaction of ad type and exposure time $\left(\chi^{2}<1\right)$ proved to be significant. Similarly, no differences were found in the total number of distinct ad items recalled, as listed in the openended ad recall measure. Specifically, an equivalent number of items was recalled for the element-absent (mean $=3.25$ ) and the element-present $($ mean $=2.95, F(1,88)<1$, n.s.) advertisements. The index of total thoughts, which provides a direct measure of the extent of ad elaboration (Petty, Cacioppo, and Schumann 1983), revealed a similar pattern. The total number of thoughts for the element-absent (mean $=2.10)$ versus the element-present $($ mean $=1.85$, $\mathrm{F}(1,88)=1.26, p>.26)$ advertisement did not differ significantly, supporting the idea that overall ad elaboration did not differ across conditions. None of the other analysis of variance effects was significant on either of these two measures.

Control advertisement results. Recall results for the control (product-unrelated element-absent) advertisement provided additional insights into the mechanism underlying the key finding of improved product category recall for the element-absent versus the element-present conditions. As discussed previously, the distinctiveness mechanism predicts a similar recall improvement for the control advertise- 
Table 1

OUTCOME MEANS: EXPERIMENT 1 (OLYMPUS CAMERAS)

\begin{tabular}{|c|c|c|c|c|c|}
\hline & \multicolumn{2}{|c|}{ Time: 8 Seconds } & \multicolumn{2}{|c|}{ Time: 4 Seconds } & \multirow{2}{*}{$\frac{\text { Time: } 8 \text { Seconds }}{\text { Control Ad }}$} \\
\hline & Complete Ad & Incomplete Ad & Complete Ad & Incomplete Ad & \\
\hline Product recall & $\begin{array}{c}.59 \\
(.50)\end{array}$ & $\begin{array}{l}.89 \\
(.32)\end{array}$ & $\begin{array}{c}.65 \\
(.49)\end{array}$ & $\begin{array}{c}.83 \\
(.38)\end{array}$ & $\begin{array}{c}.36 \\
(.49)\end{array}$ \\
\hline Brand recall & $\begin{array}{c}.23 \\
(.43)\end{array}$ & $\begin{array}{c}.26 \\
(.45)\end{array}$ & $\begin{array}{c}.30 \\
(.47)\end{array}$ & $\begin{array}{c}.13 \\
(.34)\end{array}$ & $\begin{array}{c}.04 \\
(.20)\end{array}$ \\
\hline Total ad recall & $\begin{array}{c}2.86 \\
(1.68)\end{array}$ & $\begin{array}{c}3.07 \\
(1.41)\end{array}$ & $\begin{array}{c}3.05 \\
(2.14)\end{array}$ & $\begin{array}{c}3.46 \\
(1.72)\end{array}$ & $\begin{array}{c}1.72 \\
(1.14)\end{array}$ \\
\hline Total ad thoughts & $\begin{array}{l}1.86 \\
(.91)\end{array}$ & $\begin{array}{c}2.07 \\
(1.03)\end{array}$ & $\begin{array}{c}1.85 \\
(1.09)\end{array}$ & $\begin{array}{c}2.12 \\
(1.11)\end{array}$ & $\begin{array}{l}1.24 \\
(.88)\end{array}$ \\
\hline
\end{tabular}

Notes: Standard deviations are in parentheses. For product recall and brand recall, the cell means represent the proportion of participants that correctly recalled the product/brand. Thus, the first number indicates that $59 \%$ of participants in the complete advertisement/ 8 -second exposure condition correctly recalled having seen an advertisement for cameras. Complete $\mathrm{ad}=$ element-present advertisement; incomplete ad = element-absent advertisement; control ad = advertisement with circular outline (exposed for 8 seconds).

ment. The specific activation mechanism, however, argues otherwise, on the grounds that the outline in the control advertisement is unlikely to induce generation (and thus activation) of a product-related node. Indeed, focusing specifically on the product-unrelated outline could detract from the attention paid to other ad elements, consequently leading to decreased overall recall, without any offsetting gains that might be obtained if product-related nodes were activated by the outline. The recall results for the control condition supported the latter line of reasoning. Only $36 \%$ of participants who were exposed to the control advertisement were able to recall the product category (cameras) correctly. Thus, not only did a product-unrelated omission not yield improved recall, but product category recall in this condition was significantly lower $\left(\chi^{2}=4.21, p<.05\right)$ than for the element-present advertisement (recall $=61.9 \%$ ). This recall advantage for the element-present advertisement compared with the control advertisement was also manifested in brand recall (element-present $=28.21 \%$, control $=4 \% ; \chi^{2}=$ $5.86, p<.05)$, as well as in the total number of distinct items recalled from the advertisement (element-present $=2.95$, control $=1.72 ; \mathrm{F}(1,64)=8.15, p<.01)$.

Follow-up study: delayed recall. The main study was run with a five-minute delay between exposure and recall measurement. However, advertisers are typically interested in long-term effects of their advertisements. Accordingly, a follow-up experiment with a two-day delay was also conducted. Subjects $(n=75)$ were exposed to either the element-absent or the element-present advertisement. Exactly the same procedure as was used in the main experiment was followed (only the 8-second exposure was studied); the only difference was that recall measures were taken two days after ad exposure. Again, element omission led to a significant improvement in product category recall (element-absent $=30.77 \%$, element-present $=8.33 \% ; \chi^{2}=$ $5.89, p<.05$ ), though not for brand recall (element-absent $=$ $5.13 \%$, element-present $=2.78 \% ; \chi^{2}<1$, n.s.) or total ad recall (element-absent $=1.00$, element-present $=1.08$; $\mathrm{F}(1,70)<1$, n.s. $)$.

\section{Discussion}

The major goal of Experiment 1 was to examine whether overt element omission would lead to any improvement in recall compared with the presence of the element in the advertisement. Our results support an affirmative answer. The omission of a central ad element, which was related to the product category, led to a significant improvement in category recall. This is a somewhat counterintuitive finding, as it might justifiably be expected that ad recall along dimensions related to a specific element would be better if the element were present in the advertisement. Instead, our results suggest that deliberate element omission can be a useful strategy from an advertiser's perspective. Indeed, given that most print advertisements are typically processed for short durations (Burnett and Moriarty 1998), advertisers might find it particularly encouraging that our results were obtained with exposure times as low as 8 and 4 seconds. Note, however, that an improvement in only product category recall, as documented in Experiment 1, might sometimes be counterproductive (e.g., for new brands, for me-too brands); Experiment 2 illustrates how the element omission strategy can be used to improve brand name recall as well.

From a theoretical perspective, our findings supported a specific activation explanation of the generation effect rather than the artifactual explanation that relies on a distinctiveness-based elaboration mechanism. An important piece of evidence against the latter process came from the control advertisement results. If ad distinctiveness (arising from the use of an outline rather than the complete element) were responsible for the improved category recall obtained for the element-absent advertisement, we would have expected a similar result for the control advertisement, which also featured an outline (albeit product-unrelated) instead of a complete visual element. Instead, consistent with expectations stemming from a specific activation perspective, category recall did not improve under this condition-in fact, poorer recall was obtained than with the element-present advertisement.

It should be noted that the circular outline might be thought to represent a more extreme form of incongruity/ distinctiveness than the camera-shaped outline (which presumably represents a more moderate form of incongruity). However, this would not detract from the argument presented previously in regard to the evidence offered against the distinctiveness-based explanation by the control advertisement results. In particular, although research on incongruity effects (Mandler 1982; Meyers-Levy and Tybout 1989) suggests that extreme and moderate levels of incon- 
gruity do differ in regard to the attitude produced (specifically, extreme incongruity leads to negative affect, whereas moderate incongruity produces positive affect), both types of incongruity lead to a similar increase in the amount of elaboration and recall compared with congruous conditions (Meyers-Levy and Tybout 1989). Therefore, if the camerashaped outline led to better ad recall because of the ad elaboration induced by a distinctive/incongruous element, similar recall improvement should have been observed for the control advertisement as well, which was not the case.

The specific nature of recall improvement produced by the product-related element-absent advertisement was also more consistent with a specific activation mechanism than with a process of distinctiveness-induced overall ad elaboration. In particular, the advantage in recall was restricted to the dimension that was directly related to the omitted element (i.e., product category recall) rather than manifested in a widespread improvement along other dimensions such as brand name and ad recall. The criticism might be made, however, that this result (in particular, the lack of an improvement in brand name recall) was aided by the choice of a relatively unfamiliar brand (Olympus). Therefore, we sought to replicate this result using a more familiar camera brand, which we identified on the basis of pretesting. We asked 19 students from our subject pool to write down the first camera brand that came to mind. The modal value $(n=$ $12,63.16 \%$ ) was Canon (the next most familiar brand was Sony at $n=2$ ). In a separate pretest with 16 students, we then found that Canon was rated as more familiar $(\mathbf{M}=5.81)$ than Olympus $\left(M=5.09 ; t_{\text {diff }}=3.75, p<.01\right)$ on a $1-7$ scale ( 1 = "not at all familiar," 7 = "very familiar"). ${ }^{4}$ Accordingly, we modified the two test advertisements (element-absent and element-present advertisements) from the main experiment, such that the brand name "Olympus" was replaced by "Canon" throughout. Furthermore, to provide a stronger test of the specific activation hypothesis (i.e., recall improvement should be obtained only for the product category, not for the brand name), we also added the brand name in a more prominent position in the two test advertisements (see Appendix A, Part IV). We then used these advertisements to replicate the two 8 -second conditions from the main study $(n=64)$, following exactly the same procedure as before. The results were similar to those obtained in the main study-element omission led to a significant improvement in product category recall (element-absent $=82.86 \%$ element-present $=58.62 \% ; \chi^{2}=4.61, p<.05$ ) but not brand name recall (element-absent $=31.40 \%$, element-present $=$ $27.59 \% ; \chi^{2}<1$ ) or total ad recall (element-absent $=2.20$, element-present $=1.98 ; \mathrm{F}<1$, n.s.). Thus, results from this study provided further evidence for the specific activation thesis.

Finally, the lack of a moderating impact of exposure time on recall in the main study also provided insights into the underlying mechanism. If the increased category recall for the element-absent advertisement was being driven by

\footnotetext{
4To collect familiarity data for Experiment 2, which studied an advertisement for Marlboro cigarettes, participants in the first pretest were also asked to indicate what brand of cigarettes came to mind first; $73.1 \%$ responded with "Marlboro." Similarly, in the second pretest, participants rated their familiarity with Marlboro, and a rating of 5.84 was obtained. Both of these figures are comparable to those obtained for Canon.
}

greater overall elaboration in this condition (because of the distinctive/unusual appearance of the advertisement), we would have expected the recall advantage of element omission to be diminished when the processing opportunity was lowered significantly (by reducing exposure time from 8 seconds to 4 seconds). That such a moderating effect of exposure time was not obtained is another piece of evidence against the distinctiveness explanation, as is the finding that the amount of overall elaboration (as indicated by total ad thoughts) did not differ across ad type. The exposure time factor ( 8 versus 4 seconds) itself did not have a significant effect on total thoughts and total recall, which is consistent with a possibility we raised previously: The exposure times chosen were so small that overall ad elaboration was low across conditions. Again, such a floor effect on elaboration levels makes it unlikely that the improvement in category recall for the element-absent versus the element-present advertisement was a result of significant overall elaboration of the former advertisement.

It should be noted that the current findings do not negate prior research that documents the positive effects of distinctiveness and ad elaboration on recall (e.g., Goodstein 1993; Houston, Childers, and Heckler 1987). We agree that recall improvement for the element-absent advertisement may also be produced if, as a result of being perceived as distinctive/ atypical, greater overall ad elaboration were obtained in this condition compared with the element-present condition. However, the current research seeks to show that such distinctiveness-induced overall ad elaboration is not required for the positive recall effects of omission to be observed, a finding that also suggests that the generation effect in cognitive psychology cannot be fully explained as an artifact of the greater distinctiveness of incomplete elements.

\section{EXPERIMENT IA: FORCED ACTIVATION}

Although the results obtained in Experiment 1 are consistent with a process of greater specific activation induced by generation of the missing element, generation was not explicitly manipulated and measured in that study. That is, unlike the practice in cognitive psychology (e.g., Jacoby 1983; Slamecka and Graf 1978), participants were not explicitly asked to complete the incomplete item. In the advertising context of interest to us, we did not want to employ such an intrusive procedure, because any improved recall obtained by explicitly asking participants to engage in a generation task is unlikely to be representative of the results that would be obtained from exposure to the ad under more realistic conditions (for a similar argument in the context of conclusion-omission, see Kardes 1988). Having established the memorial consequences of element omission in a relatively natural setting, however, we next examined the effect of explicitly asking subjects to generate the missing element in the element-absent advertisement (versus identifying the complete element in the element-present advertisement). Strong support for the activation mechanism proposed for our previous findings would be provided if a similar pattern of results (i.e., better category recall for the element-absent advertisement) was obtained using this direct procedure.

As a secondary goal, Experiment $1 \mathrm{~A}$ also sought to replicate and extend prior advertising research on the effects of 
incomplete stimuli by investigating attitudinal consequences. Research on the effects of object cropping (Peracchio and Meyers-Levy 1994) has shown that improved brand attitudes can result from the psychological satisfaction obtained from "closing" a perceptually incomplete figure. Although object cropping differs somewhat from the types of element omission considered here (the present research focuses on cases in which the entire element is missing, not just part of its outline), the act of generating the missing element should result in the same sort of "closure" as that attained by completing a cropped object. An improvement in brand attitudes (and ad attitudes) should therefore be obtained for the element-absent versus the element-present advertisement.

\section{Procedure}

Participants $(n=45)$ were exposed to either the elementpresent or the element-absent advertisement for Olympus (the control advertisement was not studied in this followup), in the context of the same filler advertisements that were used in the main study. However, some crucial procedural changes were made. All participants were first told that they would be asked to write down answers to questions about various print advertisements that would be shown on the OHP. A seven-page booklet containing a question (one on each page) about each advertisement to be shown was then handed out. Immediately after putting up each advertisement on the OHP, the experimenter asked participants to read the question for that advertisement (questions were numbered according to the serial number of the advertisement that was shown) and write the answer to the question in the space provided in the booklet. To provide participants with sufficient time to read the question, look at the advertisement, and write the answer to the question, each advertisement was put up for 12 seconds. At the end of this time, participants were asked to turn to the next page in the booklet, and the next advertisement was put up. The question that was asked about each advertisement typically required only a one-word answer (e.g., for a filler advertisement for Clorox detergent, the question was, "What was the color of the bottle shown in the ad?"). Questions about the filler advertisements were kept the same for both element-present and element-absent conditions. The question that was asked about the test advertisement (the second to be shown) was, "In this ad, what object is (represented by the outline) hanging on the man's chest?" in the element-present (elementabsent) condition. In both cases, the correct answer that participants were expected to respond with was "camera."

After participants had responded to questions about all seven advertisements, they took part in a completely unrelated filler study that lasted approximately 30 minutes. 5 Subsequently, questionnaire booklets containing the dependent measures were handed out. In addition to the measures collected in Experiment 1 (category recall, brand recall, ad recall, ad thoughts), participants were also asked

5The delay period in both follow-up studies (Experiments IA and 2A) was fixed on the basis of logistical constraints-these studies were conducted as part of a sequence of studies run by other researchers. As a result, there was little flexibility in regard to time-related procedural details such as the duration of the filler study. to indicate their brand attitudes toward Olympus (on three seven-point semantic differential scales: "dislike"/“like," "negative"/"positive," "bad"/"good"; alpha = .86) and attitude toward the test advertisement for Olympus they had seen earlier (two seven-point semantic differential scales: "dislike"/"like," "bad"/"good"; r = .79).

\section{Results and Discussion}

All participants in the element-present condition and all but one of the participants in the element-absent condition (proportion $=1 / 22=.04$ ) wrote down the correct answer in response to the question asked about the test advertisement. Following the convention used in the generation literature (Nairne and Widner 1988; Slamecka and Graf 1978; Slamecka and Katsiati 1987), this participant was excluded from the data set. We conducted analyses on the remaining data $(n=44)$, though results remained unchanged when we included the additional participant in the data set. We expected better category recall for the element-absent advertisement (in which a camera was generated by participants) than for the element-present advertisement (in which participants focused on the complete camera hanging on the man's chest). Results supported the predictions: We observed better product category recall for the elementabsent condition (element-absent $=80.95 \%$, elementpresent $=52.17 \% ; \chi^{2}=4.05, p<.05$ ). However, the two conditions did not differ in terms of either brand recall (element-absent $=23.81 \%$, element-present $=21.74 \% ; \chi^{2}<$ 1 , n.s.) or total ad recall (element-absent $=2.16$, elementpresent $=2.43 ; \mathrm{F}(1,40)<1$, n.s. $)$. As in the main experiment, the element-absent advertisement did not receive greater overall elaboration than the element-present advertisement; the total number of ad thoughts did not differ across the two conditions (element-absent $=1.79$, elementpresent $=2.00 ; \mathrm{F}(1,40)<1$, n.s.). The pattern of ad and brand attitudes was also examined. We obtained better attitudes for the element-absent $\left(A_{a d}=4.45, A_{b r a n d}=5.00\right)$ than the element-present $\left(A_{a d}=3.84, A_{\text {brand }}=4.39 ; p<.05\right)$ advertisement. These attitude findings show that the advantage of element omission holds not just for recall but also for brand and ad attitudes, thus supporting prior persuasion findings in the object-cropping area (Peracchio and MeyersLevy 1994).

More important for the current focus, the recall results in this experiment completely replicated those obtained in the main study. Specifically, we documented an improvement in product category recall (but not in brand or ad recall) for the element-absent advertisement when participants were explicitly instructed to generate the absent element. The similarity of these findings with those in Experiment 1 suggests that the recall results in that study were indeed due to spontaneous self-generation of the missing element. We note also that subjects who were exposed to the elementpresent advertisement in the current study were explicitly asked to attend to and identify the complete camera. That significant recall improvement was still obtained for the element-absent advertisement further rebuts the possible alternative explanation that, because the complete camera in the element-present condition is less distinctive than the outline in the element-absent case, subjects simply pay it insufficient attention and display poorer category recall as a consequence. 


\section{EXPERIMENT 2}

In Experiment 2, we extend our findings in two ways. First, we examine whether the recall results obtained thus far extend to other types of element omission as well. As discussed in the introduction, instead of overtly highlighting an omission, advertisers may sometimes use a strategy of implicit omission. For example, rather than outline the missing element within an advertisement, advertisers may rely on existing ad schemas (Goodstein 1993; Goodstein, Moore, and Cours 1992) to induce the sense of an omission. Consider a hypothetical case: Advertisements for Marlboro cigarettes have traditionally featured the Marlboro cowboy, and consumers have come to expect this element in Marlboro advertisements. However, as indicated previously, legislation in the EEC bars the use of actual characters (e.g., the Marlboro Man, Joe Camel) in cigarette commercials, and similar legislation has been contemplated in the United States. If an advertisement for Marlboro left out the cowboy, the viewer might notice the absence of this ad element (on the basis of prior knowledge of Marlboro's advertising), even without the omission being highlighted by the advertiser; thus, an implicit omission can be said to have occurred.

Experiment 2 focuses on the effects of such implicit omission, extending our theorizing and findings from Experiment 1. As previously, the self-generation literature presents a rationale for the improved recall that may be obtained from such omission. Thus, in the Marlboro example, even if the cowboy is omitted, being exposed to other typical features of a Marlboro advertisement (e.g., a horse, a pack of cigarettes) can lead consumers to think of the cowboy of their own volition. Such voluntary retrieval (compared with actual exposure to the cowboy) should lead to greater activation of the cowboy concept in semantic memory and therefore positively influence later recall.

Second, in addition to examining a case of implicit omission, Experiment 2 seeks to document that the recall advantages resulting from element omission can extend to brand name recall. According to the specific activation perspective, element omission should improve recall along all dimensions that are conceptually related to the omitted element. Thus, if the omitted element is related to the brand name (instead of just the product category, as in Experiment 1), an improvement should be obtained for brand recall. In particular, for the example discussed previously, the cowboy concept is well associated with the brand name Marlboro (Assael 1998). Consequently, we would expect improved brand name recall for a Marlboro advertisement that omits the cowboy. Better category recall (but not better overall ad recall) should be obtained as well, because the cowboy is also indirectly associated with the product category of cigarettes (through the brand name).

As with overt omission, we tested these predictions using both a nonintrusive procedure in which self-generation is inferred (Experiment 2) and a more direct one (Experiment $2 \mathrm{~A}$ ) in which subjects are explicitly instructed to generate the missing element.

\section{Test Advertisement and Pretests}

An advertisement for Marlboro cigarettes was used as the test advertisement. The foreground of the element-present version featured a cowboy sitting on a horse, holding the reins (Appendix B, Part I). In addition, the advertisement consisted of the following major elements: (1) a bright orange sky; (2) in the distance, a corral with an indistinct cluster of horses; (3) an ad slogan in big white lettering, "Come to Marlboro Country," in the top portion of the advertisement; and (4) a small pack of Marlboro cigarettes under the slogan. To create the element-absent version of this advertisement, Photoshop software was used to delete the cowboy, so that the horse was featured on its own in the foreground (Appendix B, Part II). All other aspects of the advertisement remained the same across conditions.

Pretesting was carried out to check whether the Marlboro man was an important part of participants' schema for Marlboro advertisements and whether participants were likely to think of a cowboy when exposed to the element-absent advertisement. First, 17 participants were asked to list all of the elements they would normally expect to see in a Marlboro advertisement. All 17 participants listed a cowboy (or equivalents, such as "Marlboro man") in their responses. The participants were then asked to write down what else they would expect to see if they saw a horse in a Marlboro advertisement (by listing the first thing that came to their minds). Again, all participants responded with "cowboy." In a second pretest, 45 participants were shown the incomplete Marlboro advertisement on an OHP. They were asked whether anything was missing from the advertisement and, if yes, to write down the missing feature. Participants were encouraged not to think for too long over their responses and were asked simply to write down the first thing that came to mind. A significant proportion ( $n=37,80.4 \%$ ) listed the Marlboro man/cowboy. Together, these pretests suggest that a cowboy is indeed an integral part of our participants' Marlboro ad schema and indicate that participants exposed to the incomplete version of the Marlboro advertisement (featuring a riderless horse) are likely to think of the cowboy on their own.

As in Experiment 1, another pretest was carried out to check that an exposure time of 8 seconds was perceived to afford greater processing opportunity than a time of $4 \mathrm{sec}$ onds. Two groups of subjects $(n=47)$ were exposed to the element-absent advertisement for either 8 or 4 seconds. They then rated whether the advertisement had been exposed for enough time to view its details, $(-4=$ "not enough time," $0=$ "just enough time," $4=$ "more than enough time"). They were also asked to indicate their agreement/disagreement with the statement, "I got enough time to view the ad details," on another nine-point scale $(1=$ "disagree," 9 = "agree"). On both scales, the 8-second exposure (Scale 1 mean $=1.16$, Scale 2 mean $=6.72$ ) was rated higher than the 4-second exposure (Scale 1 mean $=-.54$, Scale 2 mean $=4.59 ; p<.05$ for both scales $)$.

\section{Design and Procedure}

As in Experiment 1, a 2 (ad type: element-absent versus element-present) $\times 2$ (exposure level: 4 versus 8 seconds) between-subjects design was used. Note that, unlike in Experiment 1, a product-unrelated, element-absent control condition was not included because, in the current experiment, the omission was supposed to be inferred on the basis of participants' existing schema for Marlboro advertising rather than explicitly highlighted. Because such a schema is likely to primarily contain elements that are in some way 
related to the Marlboro advertising theme, it would have been difficult to create an implicit omission that was wholly unrelated to the product/brand.

The procedure was the same as in Experiment 1 (including use of the same filler advertisements), except that participants $(n=146)$ returned to the lab two days after ad exposure to complete what they thought would be an unrelated study. At that time, the experimenter reminded participants that they had seen some advertisements two days earlier and then administered the questionnaire containing the dependent variables. A longer delay was used than in Experiment 1 to prevent possible ceiling effects arising from subjects' high familiarity with Marlboro advertising. As in Experiment 1, ancillary dependent variables (related to age, sex, and brand familiarity) did not affect any of the obtained results.

\section{Results}

Given the relatedness of the missing element (cowboy) to the brand name and therefore the product category, the specific activation mechanism predicts a recall advantage for the element-absent advertisement along both of these dimensions, in contrast to our previous study in which such an advantage was obtained only for product category recall. Analyses yielded supportive results. Element omission produced better product category recall (element-absent recall proportion $=68.35 \%$, element-present recall proportion $=$ $50.00 \% ; \chi^{2}=5.41, p<.05$ ), as well as better brand recall (element-absent $=67.08 \%$, element-present $=45.70 \% ; \chi^{2}=$ $7.46, p<.01)$. Furthermore, ad type did not interact with exposure level for either brand or category recall $\left(\chi^{2}<1\right.$ in each case), which shows that the recall advantage for the element-absent (versus element-present) advertisement persisted irrespective of whether participants were exposed to the test advertisement for 8 seconds or 4 seconds (for cell proportions, see Table 2). As in Experiment 1, this pattern of results is more consistent with the relatively effortless specific activation mechanism (which requires activation of only a single element) than with a process of overall ad elaboration.

Analyses were also conducted on the total number of pictorial and text items recalled from the advertisement as well as on the total number of ad-relevant thoughts. No significant results were obtained on either of these measures. In particular, the element-absent advertisement did not lead to greater total recall $($ mean $=2.67$ ) or greater total thoughts (mean $=1.85)$ than did the element-present advertisement (mean $=2.97,1.92 ; \mathrm{F}<1$ ). Again, these findings are consistent with the specific activation mechanism and argue against the idea that element omission led to greater overall elaboration of the advertisement. ${ }^{6}$ Finally, an examination of thought content showed that none of the participants in the element-absent condition made any mention of finding the advertisement unusual or incongruent.

A post hoc analysis of the specific content of participants' open-ended recall data offered an interesting, albeit nonconclusive, perspective on the notion that subjects spontaneously generated the cowboy in the element-absent condition. Specifically, we coded the recall data to examine whether participants remembered seeing a cowboy (or equivalents, such as "man," "man on horse," and so forth) in the advertisement. As would be expected, a high proportion of participants $(51$ of $69,73.91 \%$ ) who were exposed to the element-present advertisement recalled seeing the cowboy. More interesting, of the participants exposed to the elementabsent advertisement, which had not featured a cowboy, a substantial proportion ( 38 of $79,48.10 \%$ ) still wrote that the advertisement had contained a cowboy. Though not conclusive in itself, this pattern is consistent with a self-generation perspective: Participants who were exposed to the incomplete advertisement generated a cowboy at exposure and later misattributed their recalling the cowboy to actually having seen it in the advertisement (see Abelson 1981). It should be noted that the generation-based explanation for the improved product and brand recall in the element-absent (versus element-present) condition does not require that participants should actually (incorrectly) remember having seen the cowboy in the element-absent condition. Improved recall should still be obtained as long as participants generate the cowboy concept during ad exposure, even if they later remain aware that they did not actually see the cowboy in the advertisement.

${ }^{6}$ Because total recall and thoughts were both measured after a two-day delay, it is possible that these checks do not reflect the overall elaboration that actually took place at the time of exposure to the ad. Therefore, we also examined the effects of exposure to the element-present and element-absent advertisements on these two outcomes in an earlier pilot study in which we measured ad recall and ad thoughts immediately after exposure. Again, we obtained no difference in either total thoughts or total recall across the two advertisement versions.

Table 2

OUTCOME MEANS: EXPERIMENT 2 (MARLBORO CIGARETTES)

\begin{tabular}{|c|c|c|c|c|}
\hline & \multicolumn{2}{|c|}{ Time: 8 Seconds } & \multicolumn{2}{|c|}{ Time: 4 Seconds } \\
\hline & Complete Ad & Incomplete Ad & Complete Ad & Incomplete Ad \\
\hline Product recall & $\begin{array}{c}.49 \\
(.51)\end{array}$ & $\begin{array}{c}.71 \\
(.46)\end{array}$ & $\begin{array}{c}.51 \\
(.51)\end{array}$ & $\begin{array}{c}.66 \\
(.48)\end{array}$ \\
\hline Brand recall & $\begin{array}{c}.49 \\
(.51)\end{array}$ & $\begin{array}{c}.74 \\
(.45)\end{array}$ & $\begin{array}{c}.43 \\
(.50)\end{array}$ & $\begin{array}{c}.61 \\
(.49)\end{array}$ \\
\hline Total ad recall & $\begin{array}{c}3.15 \\
(1.86)\end{array}$ & $\begin{array}{c}2.54 \\
(1.07)\end{array}$ & $\begin{array}{c}2.80 \\
(1.49)\end{array}$ & $\begin{array}{c}2.80 \\
(1.23)\end{array}$ \\
\hline Total ad thoughts & $\begin{array}{l}1.82 \\
(.95)\end{array}$ & $\begin{array}{c}1.70 \\
(1.13)\end{array}$ & $\begin{array}{c}2.03 \\
(1.10)\end{array}$ & $\begin{array}{l}1.97 \\
(.82)\end{array}$ \\
\hline
\end{tabular}

Notes: Standard deviations are in parentheses. For product recall and brand recall, the cell means represent the proportion of participants that correctly recalled the product/brand. Thus, the first number indicates that $49 \%$ of participants in the complete advertisement/8-second exposure condition correctly recalled having seen an advertisement for cigarettes. Complete ad = element-present advertisement; incomplete ad = element-absent advertisement. 


\section{Experiment 2A: Forced Activation}

Overview and procedure. As with overt omission, a follow-up study was also run to provide more direct support for the posited generation mechanism underlying the obtained recall effects. The procedure followed was similar to that used in Experiment 1A. Participants in two different conditions (element-absent versus element-present test advertisement) wrote down answers to questions about each of seven advertisements. The question that was asked about the Marlboro test advertisement (the second to be shown in both conditions) was, "In this ad, who is (who would you usually expect to see) sitting on the horse?" in the elementpresent (element-absent) condition. In both conditions, variants of "cowboy" (e.g., "Marlboro man") were coded as correct. As in Experiment $1 \mathrm{~A}$, the key dependent measures, comprising recall and attitudes, were taken after a filler task of 30 minutes.

Results. Only one of the participants in the elementpresent condition (proportion $=1 / 31=.03$ ) and two of the participants in the element-absent condition (proportion = $2 / 30=.06$ ) failed to write down the correct answer in response to the question about the test advertisement. Analyses were conducted on all the remaining participants $(n=58)$, though results remained unchanged when all participants were included. We expected an improvement in brand name as well as category recall for the element-absent advertisement compared with the element-present advertisement, though no improvement was expected for total ad recall. As expected, element omission led to better product category recall (element-absent $=82.16 \%$, elementpresent $=53.33 \% ; \chi^{2}=5.45, p<.05$ ), as well as better brand recall (element-absent $=75.00 \%$, element-present $=$ $50.00 \% ; \chi^{2}=3.84, p<.06$ ). Total ad recall did not differ across the two conditions (element-absent $=2.71$, elementpresent $=2.39 ; F(1,54)<1$, n.s. $)$, nor did the total number of ad thoughts (element-absent $=1.53$, element-present $=$ $1.25 ; F(1,54)=1.92, p>.17)$. Finally, in contrast to findings in Experiment $1 \mathrm{~A}$, ad and brand attitudes also did not differ for the element-absent advertisement $\left(A_{a d}=4.55\right.$, $A_{b r a n d}=3.31$ ) versus the element-present advertisement $\left(A_{\mathrm{ad}}=4.73, A_{\text {brand }}=3.17 ; \mathrm{F}<1\right)$. Possible explanations for this null finding are discussed subsequently.

\section{Discussion}

Similar to overt element omission, implicit element omission was found to lead to improved recall. Furthermore, unlike Experiment 1, in which this improvement was manifested only for category recall, Experiment 2 (as well as the follow-up Experiment 2A) also documented an advantage in brand recall. This finding is of interest to marketers, because merely improving category recall may not always be desirable (indeed, this can be counterproductive, especially for low-share brands). By showing that the omission of a brand-related feature can lead to an improvement in brand recall, our findings offer suggestions for more specific omission strategies that may be employed by advertisers and marketers.

From a public policy perspective, these results suggest that policymakers must be careful to avoid simplistic/legalistic efforts to ban the use of well-known advertising characters (such as the Marlboro Man). This approach may not work, at least in the short run, as long as the target audience maintains the initial schema that includes the banned feature. Indeed, if the original schema is maintained, there is the potential for forced element omission to result in a more, rather than less, effective ad campaign in terms of later recall.

The findings from Experiment 2 are again more consistent with the specific activation view of the generation effect, rather than the alternative view that posits that improved recall is simply an artifact of the greater overall processing that is allocated to a distinctive stimulus (in this case, a distinctive advertisement). Consistent with the former mechanism, we obtained a recall advantage of element omission along the specific dimensions related to the missing element, rather than the overall improvement in ad recall that would be predicted by the latter. Furthermore, we found that a reduction in processing opportunity (from 8 seconds to 4 seconds) did not detract from the recall advantages for the element-absent condition; again, this finding is more consistent with the relatively effortless specific activation mechanism than with an effortful process of overall elaboration. Finally, findings in Experiment $2 \mathrm{~A}$, in which generation of the missing element was manipulated and measured, converged with those obtained in the main study, further bolstering the specific activation perspective.

Experiment 2A (unlike Experiment 1A) found no attitude differences for the element-absent advertisement versus the element-present advertisement. There could be several reasons for this difference in results between the two experiments. For example, it might well be that attitudes toward Marlboro are entrenched and possibly more extreme (because of strong feelings about the product category and/ or the brand) than equivalent attitudes toward Olympus cameras; therefore, the satisfaction obtained as a result of closure may not be enough to influence existing attitudes toward Marlboro. Another explanation (for which we thank an anonymous reviewer) involves possible differences between different types of omission. Specifically, it seems plausible that viewers perceive the omission in the case of the Olympus advertisement as more creative and clever than the omission in the Marlboro advertisement, and therefore the former is more likely to translate to better attitudes than the latter. Thus, an attitude improvement may be obtained only for relatively creative omissions, which not only allow viewers the satisfaction of closure but also motivate viewers to reward the advertisers for their creativity. It would be interesting to investigate this conjecture in further research.

\section{GENERAL DISCUSSION}

The major goal of this research was to investigate the memory consequences of element omission in advertising. Findings documented for two different types of omissions indicate that significant recall advantages can accrue from this strategy. Our results carry straightforward implications for advertisers and marketers: Not only do they show that the omission of an element can increase recall, but they also offer guidelines as to the dimensions along which recall improvement will be obtained. Thus, omitting an element that is related to the product category but not to a specific brand results only in improved category recall; however, if the omitted element is related to the brand as well, a brand recall advantage can also be obtained.

We note that these recall effects were obtained with exposure times as low as 4 seconds. It therefore appears that very 
little processing may sometimes be required for important consequences to be produced. This observation is consistent with recent work in social cognition by Ambady, Bernieri, and Richeson (2000), which suggests that substantial processing effects can arise from very "thin slices" of processing. Whereas Ambady, Bernieri, and Richeson focus on judgment effects that might be obtained with thin-slice processing, the current research extends this premise to the domain of recall effects. In a more applied vein, the notion that a strategy of element omission can improve recall through relatively effortless routes is of particular value to the advertiser, because consumers typically have little motivation and/or opportunity to process advertisements carefully (Burnett and Moriarty 1998; Kassarjian 1978; Krugman 1965). Conversely, policymakers may wish to consider the potentially adverse consequences of our findings. Forcing advertisers to omit ad elements that have long been part of their advertising (e.g., the Marlboro Man, Joe Camel) may actually improve consumers' memory for these elements.

Although the primary focus of our research was recall effects of omission, some of our findings (Experiment 1A) show that the positive effects of omission can extend to attitudes as well. From the advertiser's perspective, therefore, the strategy of omission potentially offers widespread advantages. We note also that, in documenting improved attitudes for the case of overt element omission, the current research replicates prior advertising work that has found evidence for the improvement in attitudes produced by a strategy of object cropping (Peracchio and Meyers-Levy 1994). We extend these findings to a new context and show that a positive attitudinal impact can be obtained for ad attitudes in addition to brand attitudes.

From a theoretical viewpoint, this article provides insights into the process underlying the generation effect. In particular, the pattern of obtained results opposes the view that the generation effect is simply an artifact arising from the greater distinctiveness of incomplete (versus complete) items. Rather, the specific nature of recall improvement documented in our main studies and the parallel results we obtained by explicitly asking participants to generate the missing element support the position that there is a substantive difference between generating an item and processing that same element when it is provided in a complete form; in particular, generation leads to greater semantic activation (and thus better recall) of that item. We also note that the current study appears to be one of the first to document the generation effect for visual omissions-existing work in this area has focused primarily on textual omissions (e.g., word fragments) in studying this effect. Given that visual and verbal information may differ in important respects in terms of memory representation and processing modes (Holbrook and Moore 1981; Smith and Magee 1980), it is reassuring that the generation effect holds for visual stimuli.

Although the results obtained in our studies are encouraging, some limitations should be noted. In particular, our use of real advertisements as the test advertisements enhanced the external validity of our findings, but we might have achieved greater experimental control by creating our own (fictitious) advertisements. Moreover, the exclusive use of student subjects (albeit a relevant population for the product categories studied here) and the use of only one test advertisement to study each type of omission limit the gen- eralizability of our findings. A replication with other samples and stimuli would be reassuring. In addition, although we use the terms "overt omission" and "implicit omission" for expository convenience, future work in this area should strive to provide a more complete typology of different types of omissions. Clearly, overt and implicit omissions differ in certain respects. An advertisement such as the elementabsent Olympus advertisement (Experiment 1) that overtly highlights the absence of a pictorial element through the outlining technique may be regarded as incomplete. However, the element-absent advertisement in Experiment 2 (in which the cowboy is not included) would probably still be considered pictorially complete. It could perhaps be argued that the difference is a quantitative rather than a qualitative one: If placed along a complete versus incomplete continuum, an advertisement featuring an overt omission would be placed more toward the incomplete end of that continuum than would an advertisement with an implicit omission. Even so, both these types of advertisements would probably be regarded as more incomplete than the complete end of the continuum, which explains the similarity in recall outcomes produced by these advertisements. However, this suggestion is a speculative one. More work is needed to provide a classification of the different types of omissions in advertising and to document similarities and differences in their effects on recall.

Future research should also strive to uncover moderators for the memory advantages of element omission that have been documented in the current investigation. A potentially interesting moderator in this context is consumers' level of prior knowledge (e.g., Kardes, Kim, and Lim 1994; Sanbonmatsu, Kardes, and Herr 1992). Thus, for example, if consumers are not knowledgeable about Marlboro advertising and do not possess a detailed ad schema for this brand, they are unlikely to generate an omitted feature (e.g., the cowboy) on exposure to the element-absent advertisement. Consequently, the memory advantages depicted in Experiment 2 should be obtained only for consumers who are highly knowledgeable about the different elements of the ad schema. Apart from knowledge, another factor that may moderate the current results is consumers' propensity to visualize and use imagery (Childers, Houston, and Heckler 1985); specifically, it seems logical to suggest that the recall advantages accruing because of visual element omission are more likely to be obtained for high visualizers than for low visualizers. The exploration of such moderating factors represents a promising avenue for further work in this area.

Finally, future investigations should also examine other types of omissions. Whereas we have investigated the effects of omitting a pictorial element, similar results should be obtained for the case of textual omission as well. For example, consider the advertisement for J\&B scotch whisky described previously, which uses the tag line "...ingle ells, ...ingle ells.... The holidays aren't the same without J\&B." Consumers will typically be able to use their knowledge of the popular Christmas song ("Jingle Bells") to generate the missing letters. Our work predicts that such self-generation should produce improved recall of the letters (J, B) and thereby the brand name (J\&B), compared with an advertisement in which the complete words are provided in the tag line. Research testing such applications would further validate the ideas presented here. 
Appendix A

ADVERTISEMENTS FOR CAMERAS

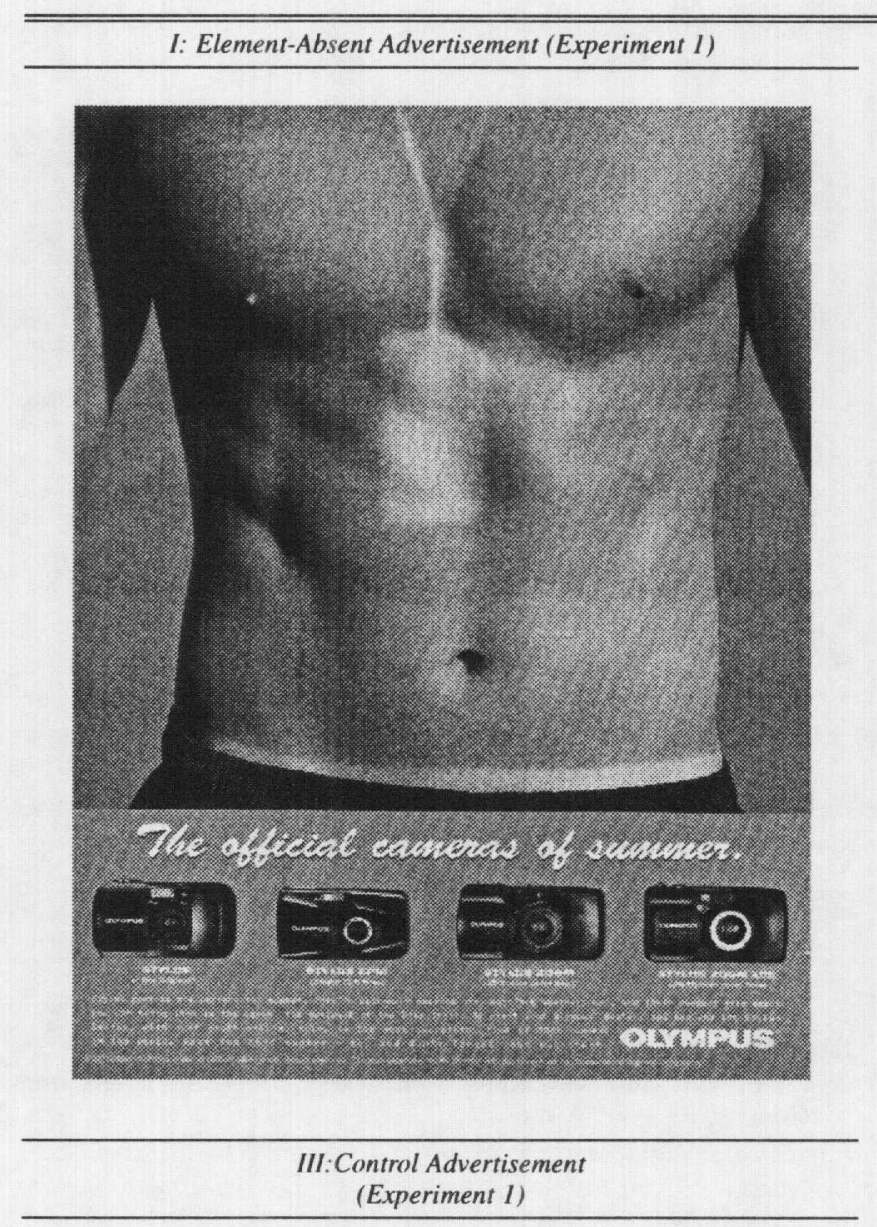

II: Element-Present Advertisement (Experiment I)

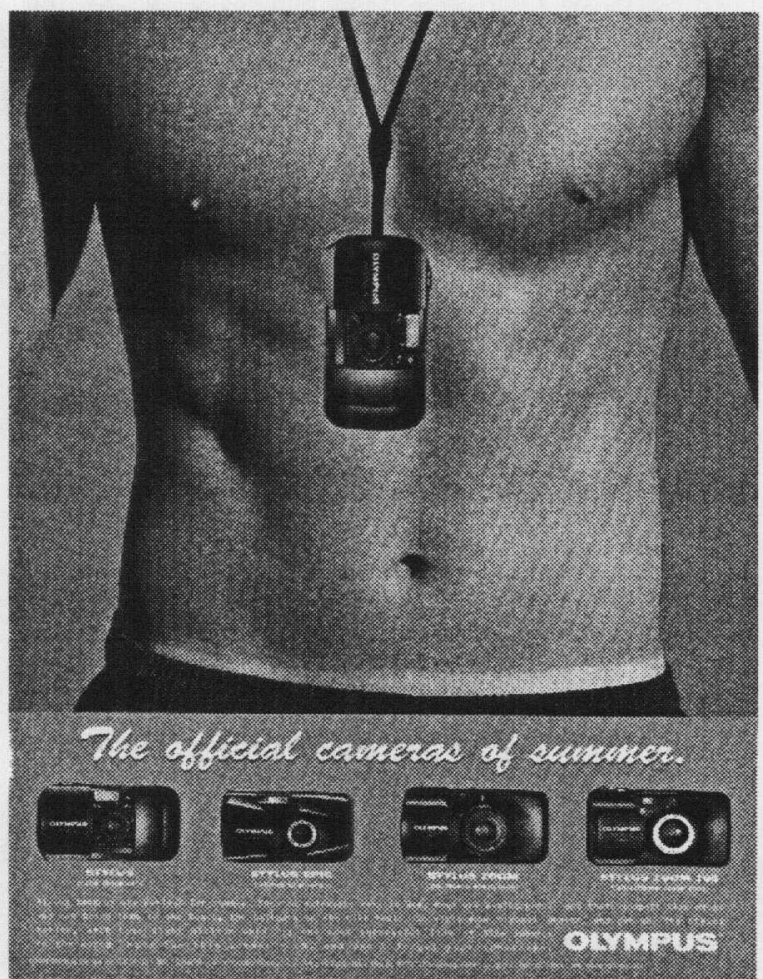

IV: Element-Absent Advertisement Featuring A Familiar Brand (Experiment 1)
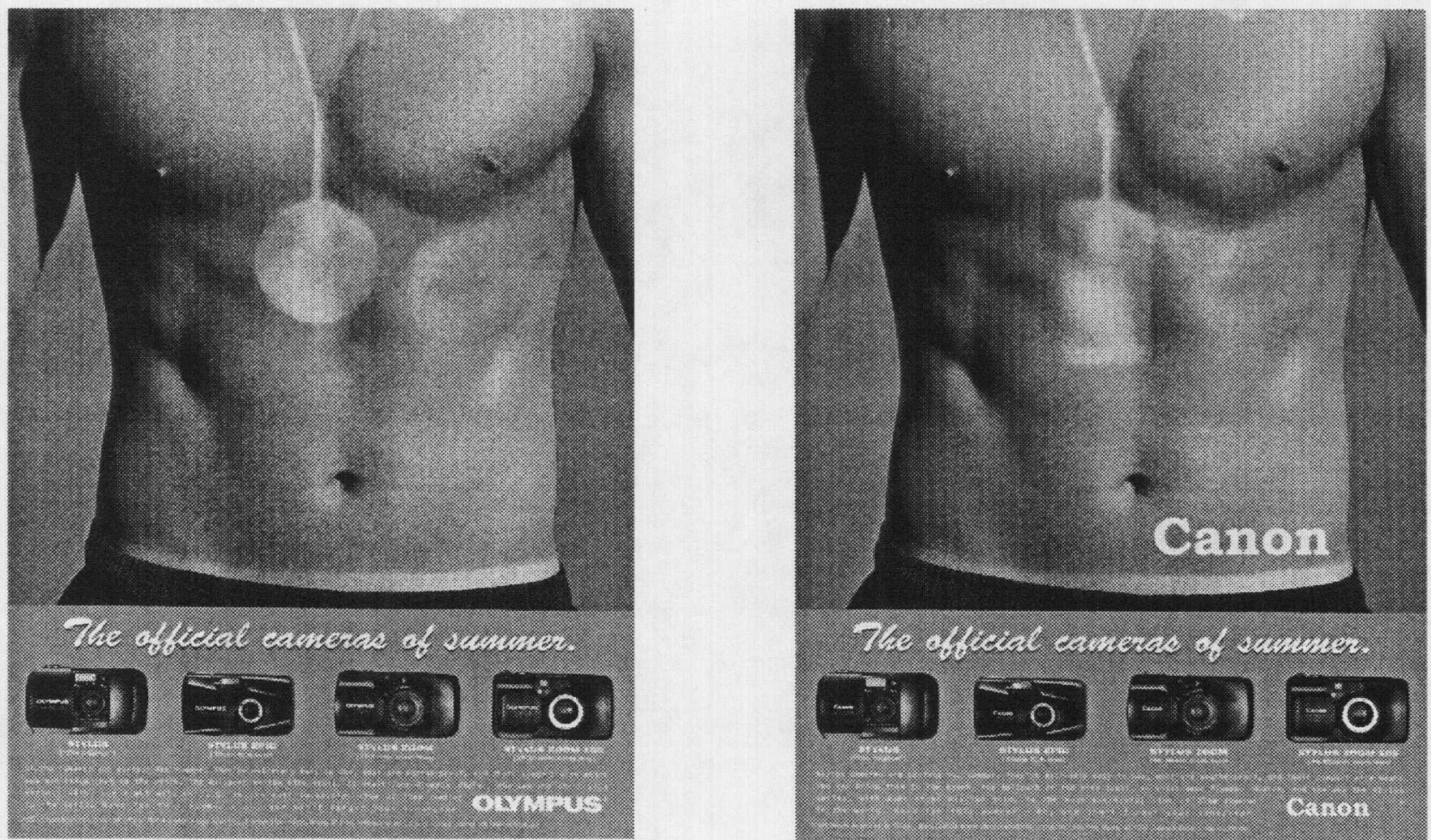
Appendix B

ADVERTISEMENTS FOR MARLBORO CIGARETTES

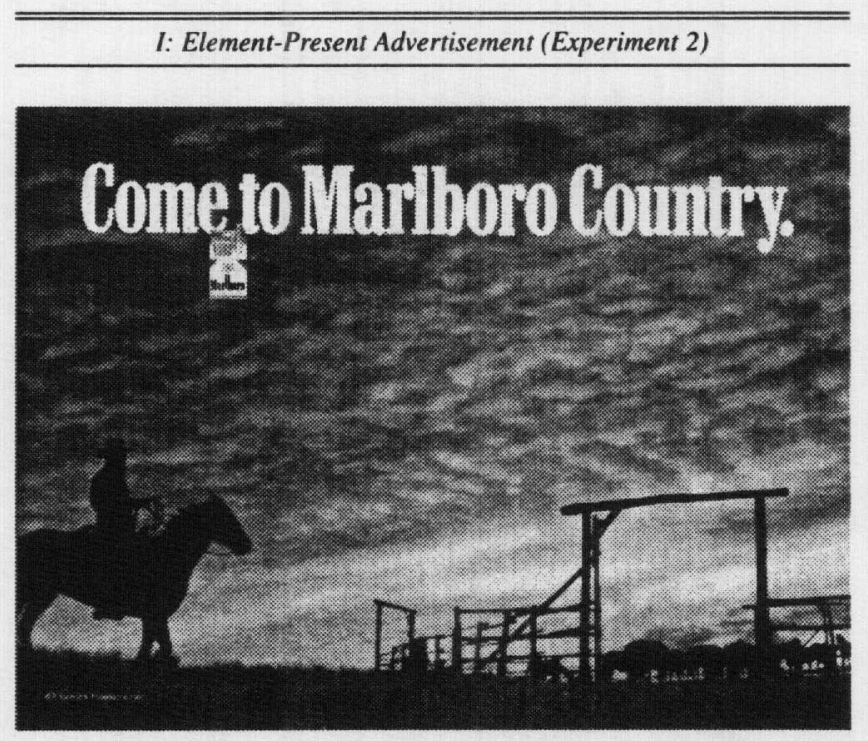

II: Element-Absent Advertisement (Experiment 2)

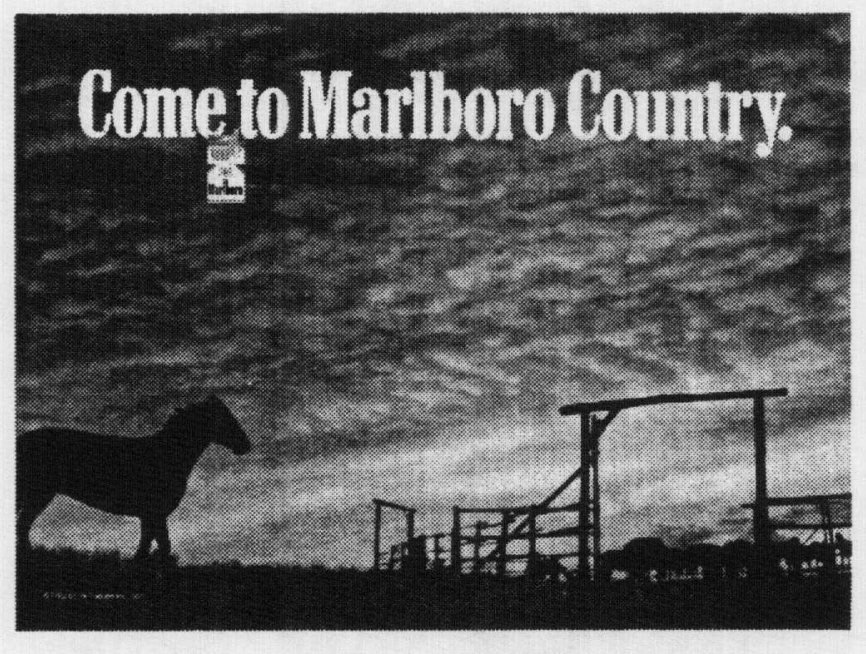

\section{REFERENCES}

Aaker, David A., Rajeev Batra, and John G. Myers (1992), Advertising Management. Englewood Cliffs, NJ: Prentice Hall.

Abelson, Robert (1981), "The Psychological Status of the Script Concept," American Psychologist, 36 (July), 715-29.

Ambady, Nalini, Frank Bernieri, and Jennifer Richeson (2000), "Toward a Histology of Social Behavior: Judgmental Accuracy from Thin Slices of the Behavioral Stream," in Advances in Experimental Social Psychology, Vol. 32, Mark P. Zanna, ed. San Diego, CA: Academic Press, 201-69.

Assael, Henry (1998), Consumer Behavior and Marketing Action. Cincinnati, OH: South-Western College Publishing.

Begg, Ian and Andrea Snider (1987), "The Generation Effect: Evidence for Generalized Inhibition," Journal of Experimental Psychology: Learning, Memory, and Cognition, 13 (4), 553-63.

Burnett, John and Sandra Moriarty (1998), Introduction to Marketing Communications. Upper Saddle River, NJ: Prentice Hall.

Childers, Terry L. and Michael J. Houston (1984), "Conditions for a Picture-Superiority Effect on Consumer Memory," Journal of Consumer Research, 11 (September), 643-54.

,$- \ldots$, and Susan E. Heckler (1985), "Measurement of Individual Differences in Visual Versus Verbal Information Processing," Journal of Consumer Research, 12 (September), 125-34.

Faier, John A. and Lynette S. Unger (1987), "The Use of Closure as a Global Advertising Technique," in The Proceedings of the 1987 Conference of the American Academy of Advertising, Florence G. Feasley, ed. Charleston, SC: The Academy, R114R117.

Gardiner, John M. (1988), "Generation and Priming Effects in Word-Fragment Completion," Journal of Experimental Psychology: Learning, Memory, and Cognition, 14 (3), 495-501.

Goodstein, Ronald C. (1993), "Category-Based Applications and Extensions in Advertising: Motivating More Extensive Processing," Journal of Consumer Research, 20 (June), 87-99.

, Marian C. Moore, and Deborah A. Cours (1992), "Exploring Advertising Schemas: A Multi-method Investigation," paper presented at the American Marketing Association Winter Educators' Conference, San Antonio, TX (February).

Heckler, Susan E. and Terry L. Childers (1992), "The Role of Expectancy and Relevancy in Memory for Verbal and Visual Information: What Is Incongruency?" Journal of Consumer Research, 18 (March), 475-92.

Holbrook, Morris B. and William L. Moore (1981), "Feature Interactions in Consumer Judgments of Verbal Versus Pictorial Presentations," Journal of Consumer Research, 8 (June), 103-13.

Houston, Michael J., Terry L. Childers, and Susan E. Heckler (1987), "Picture-Word Consistency and the Elaborative Processing of Advertisements," Journal of Marketing Research, 24 (November), 359-69.

Jacoby, Larry L. (1983), "Remembering the Data: Analyzing Interactive Processes in Reading," Journal of Verbal Learning and Verbal Behavior, 22, 485-508.

Johar, Gita Venkataramani (1995), "Consumer Involvement and Deception from Implied Advertising Claims," Journal of Marketing Research, 32 (August), 267-79.

Kardes, Frank R. (1988), "Spontaneous Inference Processes in Advertising: The Effects of Conclusion Omission and Involvement on Persuasion," Journal of Consumer Research, 15 (September), 225-33.

- John Kim, and Jeen-Su Lim (1994), “Moderating Effects of Prior Knowledge on the Perceived Diagnosticity of Beliefs Derived from Implicit Versus Explicit Product Claims," Journal of Business Research, 29 (March), 219-24.

Kassarjian, Harold H. (1978), "Presidential Address, 1977: Anthromorphism and Parsimony," in Advances in Consumer Research, Vol. 5, Keith Hunt, ed. Ann Arbor, MI: Association for Consumer Research, xii-xiv.

Kreitler, Hans and Shulamith Kreitler (1972), Psychology of the Arts. Durham, NC: Duke University Press.

Krugman, Herbert E. (1965), "The Impact of Television Advertising: Learning Without Involvement," Public Opinion Quarterly, 29 (Fall), 349-56.

Mandler, George (1982), "The Structure of Value: Accounting for Taste," in Affect and Cognition, Margaret Clark and Susan Fiske, eds. Hillsdale, NJ: Lawrence Erlbaum Associates, 3-36.

McDaniel, Mark A., Paula J. Waddill, and Gilles O. Einstein (1988), "A Contextual Account of the Generation Effect: A Three-Factor Theory," Journal of Memory and Language, 27 (October), 521-36.

McElroy, Lori A. and Norman J. Slamecka (1982), "Memorial Consequences of Generating Nonwords: Implications for Semantic-Memory Interpretations of the Generation Effect," Journal of Verbal Learning and Verbal Behavior, 21, 249-59.

McFarland, Carl E., Jr., Trudy J. Frey, and Deborah D. Rhodes (1980), "Retrieval of Internally Versus Externally Generated 
Words in Episodic Memory," Journal of Verbal Learning and Verbal Behavior, 19, 210-25.

Meyers-Levy, Joan and Alice M. Tybout (1989), "Schema Congruity as a Basis for Product Evaluation," Journal of Consumer Research, 16 (June), 39-54.

Mitchell, Andrew A. (1983), "The Effects of Visual and Emotional Advertising: An Information-Processing Approach," in Advertising and Consumer Psychology, L. Percy and A. Woodside, eds. Lexington, MA: Lexington Books, 197-217.

Nairne, James S., C.P. Pusen, and Robert L. Widner Jr. (1985), "Representation in the Mental Lexicon: Implications for Theories of the Generation Effect," Memory \& Cognition, 13 (March), 183-91.

and Robert L. Widner Jr. (1988), "Familiarity and Lexicality as Determinants of the Generation Effect," Journal of Experimental Psychology: Learning, Memory, and Cognition, 14 (4), 694-99.

Payne, David G., James H. Neely, and Daniel J. Burns (1986), "The Generation Effect: Further Tests of the Lexical Activation Hypothesis," Memory \& Cognition, 14 (3), 246-52.

Peracchio, Laura A. and Joan Meyers-Levy (1994), "How Ambiguous Cropped Objects in Ad Photos Can Affect Product Evaluations," Journal of Consumer Research, 21 (June), 190-204.

Petty, Richard E., John T. Cacioppo, and David Schumann (1983), "Central and Peripheral Routes to Advertising Effectiveness: The Moderating Role of Involvement," Journal of Consumer Research, 10 (September), 135-46.

Ray, Michael L. (1982), Advertising and Communication Management. Englewood Cliffs, NJ: Prentice Hall.

Sanbonmatsu, David M., Frank R. Kardes, and Paul Herr (1992), "The Role of Prior Knowledge and Missing Information in Mul- tiattribute Evaluation," Organizational Behavior and Human Decision Process, 51 (1), 76-91.

SAS Inc. (1999-2000), SAS System for Windows, Version 8. Cary, NC: SAS Inc.

Sawyer, Alan G. and Daniel J. Howard (1991), "Effects of Omitting Conclusions in Advertisements to Involved and Uninvolved Audiences," Journal of Marketing Research, 28 (November), 467-74.

Schacter, Daniel L. (1987), "Implicit Memory: History and Current Status," Journal of Experimental Psychology: Learning, Memory, and Cognition, 13 (3), 501-18.

Schmidt, Stephen R. and Katie Cherry (1989), "The Negative Generation Effect: Delineation of a Phenomenon," Memory and Cognition, 17 (3), 359-69.

Slamecka, Norman J. and Peter Graf (1978), "The Generation Effect: Delineation of a Phenomenon," Journal of Experimental Psychology: Human Learning and Memory, 4 (6), 592-604.

and Lilly T. Katsiati (1987), "The Generation Effect as an Artifact of Selective Displaced Rehearsal," Journal of Memory and Language, 26 (December), 589-607.

Smith, M.C. and L.E. Magee (1980), "Tracing the Time Course of Picture Word Processing," Journal of Experimental Psychology: General, 109 (December), 373-92.

Srull, Thomas K. (1981), "Person Memory: Some Tests of Associative Storage and Retrieval Models," Journal of Experimental Psychology: Human Learning and Memory, 7 (6), 440-63.

Stayman, Douglas M. and Frank R. Kardes (1992), "Spontaneous Inference Processes in Advertising: Effects of Need for Cognition and Self-Monitoring on Inference Generation and Utilization," Journal of Consumer Psychology, 1 (2), 125-42. 